\title{
Exergaming to improve physical, psychological and cognitive health among home office workers: A COVID-19 pandemic commentary
}

\author{
Hassan Sadeghi ${ }^{\mathrm{a}, \mathrm{b}, *}$ and Deborah A. Jehu ${ }^{\mathrm{c}, 1}$ \\ ${ }^{a}$ Department of Biomechanics and Sports Injuries, Faculty of Physical Education and Sports Sciences, Kharazmi \\ University, Tehran, Iran \\ ${ }^{\mathrm{b}}$ Shiraz, Geriatric Research Center, Shiraz University of Medical Sciences, Shiraz, Iran \\ ${ }^{\mathrm{c}}$ Interdisciplinary Health Sciences Department, College of Allied Health Sciences, Augusta University, Augusta, \\ GA, USA
}

Received 29 June 2021

Accepted 3 August 2021

\begin{abstract}
.
BACKGROUND: The COVID-19 pandemic has resulted in increased sedentary behaviour and poorer health among office workers. Exergaming is a technology-driven mode of exercise that can improve health while physically distancing.

OBJECTIVE: The purpose of this commentary was to explain the benefits of exergaming on physical function, psychological health, and cognition among office workers.

RESULTS: Exergaming improves these health outcomes, reduces pain, and decreases the risk for chronic disease. It is easily accessible on smart devices and can be performed both indoors and outdoors.

CONCLUSIONS: Twenty-one minutes of exergaming per day can improve health outcomes and reduce the risk of pain and disease. Employers and policy-makers should consider promoting exergaming among office workers.
\end{abstract}

Keywords: Exercise, video games, sedentary behaviour, middle-age, COVID-19

\section{Introduction}

The World Health Organization (WHO) declared the COVID-19 outbreak as a pandemic in March 2020. As of 21 July 2021, there have been over

\footnotetext{
${ }^{1}$ ORCID: https://orcid.org/0000-0002-9084-7445.

*Address for correspondence: Hassan Sadeghi, Department of Biomechanics and Sports Injuries, Faculty of Physical Education and Sports Sciences, Kharazmi University, Tehran, Iran. E-mail: hassan.sadeghi81@yahoo.com; ORCID: https://orcid.org/00000002-5602-1754
}

191 million confirmed cases and over 4.1 million deaths, posing an unprecedented challenge to alleviate this public health concern [1]. Physical inactivity has been reported to be $30 \%$ higher during the pandemic [2]. It is possible that the government-imposed restrictions (e.g., stay-at-home orders, travel restrictions, banning outdoor activity) [3], gym closures [4], delays in manufacturing of fitness equipment [4], financial hardship [5, 6], and the lack of social support from training partners [4] may have contributed to this increased physical inactivity. The consequences 
of the pandemic have therefore posed challenges to adhering to the recommended physical activity guidelines (i.e., 150 minutes per week of moderateintensity physical activity or 75 minutes per week of moderate vigorous-intensity physical activity [7]). To this end, researchers have observed negative impacts on health outcomes, such as poorer quality of life [8], loneliness [9], isolation [8], boredom [9], poorer mental health [9-11], poorer sleep [2], weight gain [2], and increased use of television, computer, and social media $[2,12]$. Therefore, improving health among office workers during and after the COVID-19 pandemic is important.

Exergaming improves health and can be performed at home regardless of restrictions [13-19]. Exergaming can be described as any kind of video game or multimedia application that requires physical movement for the purpose of controlling the game [20]. It combines smart technologies, exercise, and cognitive stimulation [21, 22]. It can be used on numerous platforms such as applications on smart phones [23], virtual reality systems [24], and consoles [25, 26]. Different types of exergames exist, including single player, as well as multi-player exergames with artificial and/or real players that are either collaborative or competitive [27-29]. Exergaming is feasible, safe, and shows high usability and acceptability in healthy and clinical populations across the lifespan [19]. To our knowledge, no research has summarized the benefits of socially distanced exergaming. The purpose of this commentary was to explain the benefits of exergaming on physical function, psychological health, and cognition among home office workers. A better understanding of the benefits of socially distanced exergaming is warranted for future uptake.

\section{Benefits of exergaming}

\subsection{Exergaming improves physical function}

Exergaming offers benefits to physical function, such as improvements in fitness, strength, energy expenditure, gait speed, posture, and functional mobility in middle aged adults relative to an inactive control group [13-17]. Multi-player exergaming promotes greater heart rate and energy expenditure compared to single-player exergaming, which may be a function of the games themselves or the social support provided during multi-player exergaming [30]. Exergaming also reduces cardiovascular risk, such as weight loss as well as lower blood pressure, total serum cholesterol, triglycerides, and low-density lipoprotein [13, 31, 32]. Lower-back pain is the most common occupational disease and is related to sedentary activity in office work [33]; importantly, exergaming decreases chronic neck and back pain among office workers [34]. These improvements in physical function following exergaming may stem from the games involving stepping and standing in place, head movements, performing high speed hand-eye and foot-eye coordination tasks, crouching, reaching, and jumping [16, 35]. Office-workers spend about two-thirds of their workday sitting; thus, increasing physical activity and physical function is especially important in this population [36].

\subsection{Exergaming improves psychological health}

Maintaining psychological health is important during the pandemic. Exergaming enhances mood [18], relieves stress [28], reduces social isolation [28], reduces loneliness [28], and improves self-esteem [37], self-confidence [37], and self-efficacy [37]. Multi-player exergaming increases emotional and social support, affords the opportunity to share in positive experiences with others, increases motivation and engagement in practice, and increases enjoyment compared to single-player exergaming $[29,38$, 39]. Interestingly, collaborative games increase social presence and intention to exercise compared to competitive games [27, 28], which may be important factors during the pandemic. Qualitative reports have noted that exergaming is "fun and varied", is "progressive", and offers a "challenge to do better" [18]. Altogether, socializing while physically distancing and receiving the benefits of exercise may be critical to maintaining psychological health during the pandemic.

\subsection{Exergaming improves cognitive function}

Exergaming improves cognition among office workers [40]. Specifically, exergaming improves executive function, inhibition, visuospatial processing, attention, spatial learning, memory, and cognitive flexibility in clinical and non-clinical populations [19, 41]. Exergaming is fast paced and has high perceptual, cognitive, and motor demands [42]. Therefore, perhaps these improvements are a function of the user responding to visual feedback, planning actions, understanding spatial constraints, and creating cognitive maps of bodily movements in relation to the 
game [20]. Cognitive skills are important in the workplace for tasks such as interpreting information, remember team goals, and sustained attention during meetings [43]. Thus, identifying the cognitive processes that are improved through exergaming and providing appropriate recommendations are critical for maintaining and improving cognitive function among office workers. To our knowledge, no studies have examined the effects of single vs multi-player exergaming on cognitive outcomes, which should be a future area of research.

\section{Practical implications}

Socially distanced physical activity has been recommended for office workers during the pandemic [36], with a diverse range of exercise types improving adherence [44]. This commentary expands upon previous recommendations for building a routine when working from home [45], and highlights that exergaming is a great mode of exercise to incorporate into an indoor or outdoor exercise regime for office workers. Exergaming is enjoyable, increases social engagement, increases productivity at work, is easily accessible, involves relatively low costs, and is a safe mode of exercise [26, 46, 47]. In order to meet the recommended physical activity guidelines of 150 minutes per week of moderate-intensity physical activity [7], only 21 minutes per day, 7 days/week of exergaming is necessary. Given that exergaming can improve physical function [13-17], psychological health [18], and cognition [43], and that these factors are important for the health and productivity of office workers, employers and policy makers should consider activity programs for office workers.

\section{Limitations}

While physical activity guidelines suggest 150 minutes per week of moderate-intensity physical activity [7], the minimum dose of exercise to provoke improvements in physical function, psychological health, and cognition may differ [48, 49]. Few studies have specifically examined the different types of exergames and single- vs multi-player exergames; more research is needed to better understand their influence on physical function, psychological health, and cognition.

\section{Conclusion}

Exergaming is safe, enjoyable, and low-cost. It can improve physical function, psychological health, and cognition, as well as reduce pain and prevent chronic disease. This is especially important for office workers during the pandemic who face greater sedentary behaviour. Incorporating exergaming activity programs for office workers should be considered by policy-makers and employers.

\section{Conflict of interest}

The authors have no conflict of interest to report.

\section{References}

[1] WHO Coronavirus (COVID-19) Dashboard [Internet]. WHO. 2021 [cited 6 June 2021]. Available from: https://covid19.who.int/

[2] Zheng C, Huang WY, Sheridan S, Sit CH-P, Chen XK, Wong SH-S. COVID-19 Pandemic Brings a Sedentary Lifestyle in Young Adults: A Cross-Sectional and Longitudinal Study. International Journal of Environmental Research and Public Health. 2020;17(17):6035.

[3] Coronavirus Pandemic (COVID-19) [Internet]. Our World in Data. 2020. Available from: https://ourworldindata.org/policy-responses-covid

[4] Nyenhuis SM, Greiwe J, Zeiger JS, Nanda A, Cooke A. Exercise and Fitness in the Age of Social Distancing During the COVID-19 Pandemic. J Allergy Clin Immunol Pract. 2020;8(7):2152-5.

[5] Spiro N, Perkins R, Kaye S, Tymoszuk U, MasonBertrand A, Cossette I, et al. The Effects of COVID-19 Lockdown 1.0 on Working Patterns, Income, and Wellbeing Among Performing Arts Professionals in the United Kingdom (April-June 2020). Frontiers in Psychology. 2021;11:594086.

[6] Miech RA, Kumanyika SK, Stettler N, Link BG, Phelan JC, Chang VW. Trends in the Association of Poverty With Overweight Among US Adolescents, 1971-2004. JAMA : the journal of the American Medical Association. 2006;295(20):2385-93.

[7] Foster C, Shilton T, Westerman L, Varney J, Bull F. World Health Organisation to develop global action plan to promote physical activity: time for action. British Journal of Sports Medicine. 2018;52(8):484-5.

[8] Barkley JE, Lepp A, Glickman E, Farnell G, Beiting J, Wiet $\mathrm{R}$, et al. The Acute Effects of the COVID-19 Pandemic on Physical Activity and Sedentary Behavior in University Students and Employees. International journal of exercise science. 2020;13(5):1326-39.

[9] Banerjee D, Rai M. Social isolation in Covid-19: The impact of loneliness. International Journal of Social Psychiatry. 2020;66(6):525-7.

[10] Giorgi G, Lecca LI, Alessio F, Finstad GL, Bondanini G, Lulli LG, et al. COVID-19-related mental health effects in the workplace: a narrative review. International 
Journal of Environmental Research and Public Health. 2020;17(21):7857.

[11] Sepúlveda-Loyola W, Rodríguez-Sánchez I, PérezRodríguez P, Ganz F, Torralba R, Oliveira D, et al. Impact of social isolation due to COVID-19 on health in older people: Mental and physical effects and recommendations. The Journal of Nutrition, Health \& Aging. 2020:1-10.

[12] Alomari MA, Khabour OF, Alzoubi KH. Changes in Physical Activity and Sedentary Behavior Amid Confinement: The BKSQ-COVID-19 Project. Risk Management and Healthcare Policy. 2020;13:1757-64.

[13] Staiano AE, Beyl RA, Hsia DS, Katzmarzyk PT, Newton RL. Twelve weeks of dance exergaming in overweight and obese adolescent girls: Transfer effects on physical activity, screen time, and self-efficacy. Journal of Sport and Health Science. 2017;6(1):4-10.

[14] Ren X, Hollander L, Marel Rvd, Molenaar L, Lu Y, editors. Step-by-step: Exploring a social exergame to encourage physical activity and social dynamics among office workers. Extended Abstracts of the 2019 CHI Conference on Human Factors in Computing Systems; 2019.

[15] Staiano AE. Exergames, Energy Expenditure, and Obesity. The International Encyclopedia of Media Psychology. 2020:1-7.

[16] Sadeghi H, Jehu DA, Daneshjoo A, Shakoor E, Razeghi M, Amani A, et al. Effects of 8 Weeks of Balance Training, Virtual Reality Training, and Combined Exercise on Lower Limb Muscle Strength, Balance, and Functional Mobility Among Older Men: A Randomized Controlled Trial. Sports Health. 2021;0(0):1941738120986803.

[17] Ditchburn J-L, van Schaik P, Dixon J, MacSween A, Martin D. The effects of exergaming on pain, postural control, technology acceptance and flow experience in older people with chronic musculoskeletal pain: a randomised controlled trial. BMC Sports Science, Medicine and Rehabilitation. 2020;12(1):63.

[18] Rosenberg D, Depp CA, Vahia IV, Reichstadt J, Palmer BW, Kerr J, et al. Exergames for Subsyndromal Depression in Older Adults: A Pilot Study of a Novel Intervention. The American Journal of Geriatric Psychiatry. 2010;18(3):2216.

[19] Stanmore E, Stubbs B, Vancampfort D, de Bruin ED, Firth $\mathrm{J}$. The effect of active video games on cognitive functioning in clinical and non-clinical populations: A meta-analysis of randomized controlled trials. Neuroscience \& Biobehavioral Reviews. 2017;78:34-43.

[20] Staiano AE, Calvert SL. Exergames for Physical Education Courses: Physical, Social, and Cognitive Benefits. Child Dev Perspect. 2011;5(2):93-8.

[21] Oh Y, Yang S, editors. Defining exergames and exergaming. Proceedings of Meaningful Play; 2010.

[22] Berkovsky S, Freyne J, Coombe M. Physical activity motivating games: be active and get your own reward. ACM Transactions on Computer-Human Interaction (TOCHI). 2012;19(4):1-41.

[23] Joo S-Y, Lee C-B, Joo N-Y, Kim C-R. Feasibility and Effectiveness of a Motion Tracking-Based Online Fitness Program for Office Workers. Healthcare. 2021; 9(5):584.

[24] Kim SYS, Prestopnik N, Biocca FA. Body in the interactive game: How interface embodiment affects physical activity and health behavior change. Computers in Human Behavior. 2014;36:376-84.

[25] Ren X, Yu B, Lu Y, Brombacher A. Exploring cooperative fitness tracking to encourage physical activity among office workers. Proceedings of the ACM on Human-Computer Interaction. 2018;2(CSCW):1-20.

[26] Rand D, Kizony R, Weiss PTL. The Sony PlayStation II EyeToy: low-cost virtual reality for use in rehabilitation. Journal of Neurologic Physical Therapy. 2008;32(4):15563.

[27] Wu Z, Li J, Theng YL. Examining the Influencing Factors of Exercise Intention Among Older Adults: A Controlled Study Between Exergame and Traditional Exercise. Cyberpsychol Behav Soc Netw. 2015;18(9):521-7.

[28] Li J, Theng Y-L, Foo S. Effect of exergames on depression: a systematic review and meta-analysis. Cyberpsychology, Behavior, and Social Networking. 2016;19(1):34-42.

[29] Kaos MD, Beauchamp MR, Bursick S, Latimer-Cheung $\mathrm{AE}$, Hernandez $\mathrm{H}$, Warburton DE, et al. Efficacy of online multi-player versus single-player exergames on adherence behaviors among children: A nonrandomized control trial. Annals of Behavioral Medicine. 2018;52(10): 878-89.

[30] O'Donovan C, Hirsch E, Holohan E, McBride I, McManus R, Hussey J. Energy expended playing Xbox Kinect ${ }^{\mathrm{TM}}$ and Wii ${ }^{\mathrm{TM}}$ games: a preliminary study comparing single and multiplayer modes. Physiotherapy. 2012;98(3):224-9.

[31] Jo E-A, Wu S-S, Han H-R, Park J-J, Park S, Cho K-I. Effects of exergaming in postmenopausal women with high cardiovascular risk: A randomized controlled trial. Clin Cardiol. 2020;43(4):363-70.

[32] Amorim MGS, de Oliveira MD, Soares DS, da Silva Borges L, Dermargos A, Hatanaka E. Effects of exergaming on cardiovascular risk factors and adipokine levels in women. The Journal of Physiological Sciences. 2018;68(5):671-8.

[33] Shariat A, Alizadeh R, Moradi V, Afsharnia E, Hakakzadeh A, Ansari NN, et al. The impact of modified exercise and relaxation therapy on chronic lower back pain in office workers: a randomized clinical trial. Journal of Exercise Rehabilitation. 2019;15(5):703.

[34] Jansen-Kosterink SM, Huis In 't Veld RMHA, Schönauer C, Kaufmann H, Hermens HJ, Vollenbroek-Hutten MMR. A Serious Exergame for Patients Suffering from Chronic Musculoskeletal Back and Neck Pain: A Pilot Study. Games for Health Journal. 2013;2(5):299-307.

[35] Li R, Polat U, Makous W, Bavelier D. Enhancing the contrast sensitivity function through action video game training. Nature Neuroscience. 2009;12(5):549-51.

[36] Shariat A, Cleland JA, Hakakzadeh A. Home-based exercises during the COVID-19 quarantine situation for office workers: A commentary. Work. 2020;66:381-2.

[37] Andrade A, Correia CK, Coimbra DR. The psychological effects of exergames for children and adolescents with obesity: a systematic review and meta-analysis. Cyberpsychology, Behavior, and Social Networking. 2019;22(11):724-35.

[38] Schneider J, Malinowski P, Watson PM, Lattimore P. The role of mindfulness in physical activity: A systematic review. Obesity Reviews. 2019;20(3):448-63.

[39] Andrade A, Cruz WMd, Correia CK, Santos ALG, Bevilacqua GG. Effect of practice exergames on the mood states and self-esteem of elementary school boys and girls during physical education classes: A cluster-randomized controlled natural experiment. PloS One. 2020;15(6):e0232392-e.

[40] Nooijen CFJ, Blom V, Ekblom Ö, Ekblom MM, Kallings LV. Improving office workers' mental health and cognition: a 3-arm cluster randomized controlled trial targeting physical activity and sedentary behavior in multi-component interventions. BMC Public Health. 2019;19(1):266. 
[41] Werner C, Rosner R, Wiloth S, Lemke NC, Bauer JM, Hauer $\mathrm{K}$. Time course of changes in motor-cognitive exergame performances during task-specific training in patients with dementia: identification and predictors of early training response. Journal of NeuroEngineering and Rehabilitation. 2018;15(1): 100 .

[42] Best JR. Exergaming in youth: Effects on Physical and Cognitive Health. Zeitschrift für Psychologie. 2013; 221(2):72-8

[43] Hunt E, Madhyastha TM. Cognitive demands of the workplace. Journal of Neuroscience, Psychology, and Economics. 2012;5(1):18.

[44] Sylvester BD, Curran T, Standage M, Sabiston CM, Beauchamp MR. Predicting exercise motivation and exercise behavior: A moderated mediation model testing the interaction between perceived exercise variety and basic psychological needs satisfaction. Psychology of Sport and Exercise. 2018;36:50-6.

[45] Lopez-Leon S, Forero DA, Ruiz-Díaz P. Recommendations for working from home during the COVID-19 pandemic (and beyond). Work. 2020;66(2):371-5
[46] Gomes GCV, Simões MdS, Lin SM, Bacha JMR, Viveiro LAP, Varise EM, et al. Feasibility, safety, acceptability, and functional outcomes of playing Nintendo Wii Fit PlusTM for frail older adults: A randomized feasibility clinical trial. Maturitas. 2018;118:20-8.

[47] Li J, Theng Y-L, Cheong WL, Hoo YF, Ngo MD. Exergames for the corporate wellness program in Singapore: An investigation of employees' acceptance via watching Kinect video. Digit Health. 2016;2:2055207616654578-.

[48] Borde R, Hortobágyi T, Granacher U. Dose-Response Relationships of Resistance Training in Healthy Old Adults: A Systematic Review and Meta-Analysis. Sports Med. 2015;45(12):1693-720.

[49] Sanders LMJ, Hortobágyi T, la Bastide-van Gemert S, van der Zee EA, van Heuvelen MJG. Dose-response relationship between exercise and cognitive function in older adults with and without cognitive impairment: A systematic review and meta-analysis. PloS One. 2019;14(1):e0210036-e. 